\title{
RNA in biological condensates
}

\section{THOMAS R. CECH}

Department of Biochemistry, BioFrontiers Institute, and Howard Hughes Medical Institute, University of Colorado Boulder, Boulder, Colorado 80309, USA

In assembling and editing this special issue of the RNA journal on the topic of RNA in Biological Condensates, I was in for a few surprises. I had not expected such an immense diversity of viewpoints and approaches. In fact, I had been initially concerned that a dozen authors would overlap too much in their perspectives, but I instead found that they each brought distinct elements to the conversation. Had I recruited an additional dozen authors, there probably would have still been little overlap! The articles you'll read in this issue span the gamut from in vitro to in vivo systems, from chemistry to biology, from nucleus to cytoplasm, and from worms and plants to patients.

The words in the title of this special issue were chosen with some thought. We include "RNA" not just because this is the RNA journal, but because it's clear that most of these membraneless compartments in cells are organized around RNA and RNA-binding proteins. Certainly, the proteins often have IDRs-intrinsically disordered domainsthat contribute to condensation, but RNA is by itself able to phase-separate in vitro and specific RNAs are enriched in various compartments in vivo.

"Biological" is included in the title to emphasize the importance of understanding function. The mere existence of a condensate may suggest functionality but is insufficient to assume it. Even for the nucleolus, long known to be the site of rRNA transcription, processing, modification, and assembly of proteins with the rRNA, it is difficult to show what the nucleolar structure provides to facilitate these processes. The same can be said of other condensates discussed in this issue, which are thought to function in transcription and translation. For most biological condensates, understanding function is still very much a work in progress.

Before I briefly mention each of the articles, let me reveal that I encouraged the authors to be provocative, to put out ambitious models-in order to stimulate testing of those models. Many of them rose to this challenge.

Kato, Zhou, and McKnight have a protein-centric view of condensates. They describe how the low complexity domains of proteins can self-associate via the formation of la-

Article is online at http://www.rnajournal.org/cgi/doi/10.1261/rna. 079051.121. Freely available online through the RNA Open Access option. bile cross- $\beta$ interactions that are structurally specific, yet reversible and therefore dynamic. Bevilacqua, Williams, Chou, and Assmann present an RNA-centric view, reminding us that the intrinsic thermodynamic, kinetic, and structural properties of RNA can explain much of condensation. Currie and Rosen are perhaps somewhere in the middle. They examine the evidence that cooperative interactions among multiple components promote liquidliquid phase separation, and they emphasize that composition and stoichiometry regulate biochemical activities within condensates.

Forman-Kay, Ditlev, Nosella, and Lee present a chemical analysis of RNA condensates and ask the provocative question of whether even a single long RNA could constitute a condensate. To what extent is a condensate defined by interactions between multiple molecules, instead of by interactions between different parts of a single molecule?

Darzacq and Tjian derive insights from their live cell single-particle imaging experiments. They propose a model in which the high effective concentrations of macromolecules in condensates allow even very weak interactions to lead to high binding site occupancy, thus mediating function in a highly dynamic manner. Also taking up the challenge to propose models, Sharp, Chakaborty, Henninger, and Young address the function of eRNAs (enhancer RNAs), which appear to account for a majority of the long noncoding RNAs in the nucleus. In their model, eRNAs facilitate the formation of a local condensate that stabilizes the binding of transcription factors and also accounts for the bursts of transcription seen at promoters.

Ouyang and Seydoux examine C. elegans nuage, condensates that assemble around the nuclei of developing germ cells and are enriched in proteins required for the biogenesis and function of sRNAs (silencing small RNAs). As such, nuage has been proposed to enhance sRNA function. The authors describe a new model in which nuage condensates balance the activity of competing sRNA pathways and limit sRNA amplification, thus protecting transcripts from dangerous runaway silencing.

(c) 2022 Cech This article, published in RNA, is available under a Creative Commons License (Attribution-NonCommercial 4.0 International), as described at http://creativecommons.org/licenses/ by-nc/4.0/. 
Three additional articles focus on condensates in the cytoplasm. Ripin and Parker propose a model in which stress granules are the RNA equivalent of misfolded proteins, with "RNA chaperones" such as RNA helicases and RNAbinding proteins serving to limit inappropriate RNA-RNA interactions and keep mRNP granule formation under control. Chen and Mayr address the question of how the components of multisubunit complexes can find each other in the crowded environment of the cell. In their model, scaffold RNA-binding proteins in a condensate transiently retain proteins that need to be assembled into the same complex, thereby facilitating their interaction. Roden and Gladfelter describe a new class of reporter RNAs to study the regulation of translation by phase separation. They alter the sequence of reporter RNAs to recruit them to specific condensates, facilitating analysis of the role of phase separation in translational regulation.

Finally, Nedelsky and Taylor explore the consequences of RNA-protein granules gone bad, losing their normal dynamics and leading to neurodegenerative diseases such as amyotrophic lateral sclerosis and frontotemporal dementia.

There's one word in the title of this issue that I've not yet described. And here, it is perhaps I who am being provocative, knowing that the field is not of one mind on this topic. I chose the word "condensate" over other terms such as "liquid-liquid phase separation" (LLPS) because it's wonderfully agnostic regarding the mechanism of formation. "Condensate" simply means that certain macromolecules are substantially concentrated within the defined region relative to its surroundings. LLPS, on the other hand, is a term taken from chemistry, and chemists would expect LLPS to be subject to a specific chemical meaning. A classic example is the phenolwater LLPS, which many of us have experienced when phenol-extracting DNA and RNA. In a phenol-water LLPS, the existence of the two separated phases is visible by their different refractive indices, can be verified by measuring the percent phenol in the phenolic phase and the percent water in the aqueous phase, and can be further tested by adding small aromatic molecules (which partition into the phenolic phase) and small hydrophilic molecules (which partition into the aqueous phase). It is easy to apply such criteria to LLPSs formed in vitro but difficult to apply them to many of the small cellular condensates described herein. This issue of RNA catches snapshots of the field at a moment in time when its scientists are still in the process of condensing around verifiable criteria for LLPS in vivo.

Special thanks to Ann Marie Micenmacher, who rescued me on multiple occasions when I was struggling to handle multiple manuscripts simultaneously. 

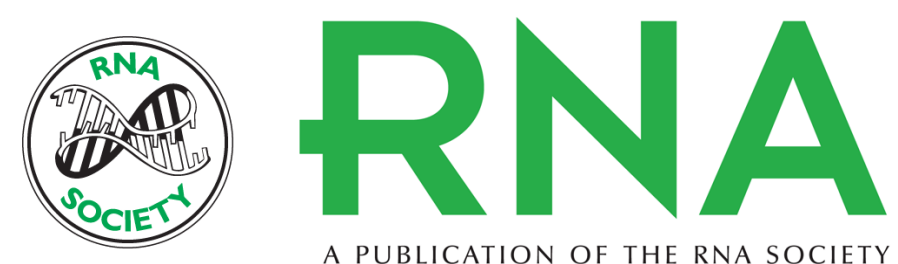

A PUBLICATION OF THE RNA SOCIETY

\title{
RNA in biological condensates
}

\author{
Thomas R. Cech
}

RNA 2022 28: 1-2
Open Access Freely available online through the RNA Open Access option.
Creative This article, published in $R N A$, is available under a Creative Commons License Commons (Attribution-NonCommercial 4.0 International), as described at License http://creativecommons.org/licenses/by-nc/4.0/. Email Alerting
Service

To subscribe to $R N A$ go to:

http://rnajournal.cshlp.org/subscriptions 